\title{
Prevalence and predictors of HIV and Selected Sexually Transmitted Infections among People Who Inject Drugs in Dar es Salaam, Tanzania: A new focus to get to zero
}

Elia John Mmbaga (MD, PhD) $)^{1,2}$, Kåre Moen (MD, PhD $)^{2}$, Neema Makyao (MPH) ${ }^{3}$, Melkizedeck Leshabari(DSc) ${ }^{4}$

\author{
Affiliations \\ ${ }^{1}$ Department of Epidemiology and Biostatistics, Muhimbili University of Health and Allied \\ Sciences, Dar es Salaam, Tanzania. \\ ${ }^{2}$ Department of Community Medicine and Global Health, Institute of Health and Society, \\ University of Oslo, Norway \\ ${ }^{3}$ National AIDS Control Programme, Ministry of Health and Social Welfare, Dar es Salaam, \\ Tanzania \\ ${ }^{4}$ Department of Behavioral Sciences, Muhimbili University of Health and Allied Sciences, Dar \\ es Salaam, Tanzania
}

\section{Name and address of corresponding author;}

Elia John Mmbaga

Department of Epidemiology and Biostatistics, Muhimbili University of Health and Allied Sciences, 9 united nations Road, P.O. Box 65015, Dar es Salaam, Tanzania. Email: eliajelia@yahoo.co.uk Telephone: +255785900101 Fax: +255-022-2150465 


\section{ABSTRACT}

\section{Background}

Previous studies in Tanzania indicated that HIV prevalence among people who inject drugs (PWIDS) could be as high as $40 \%$. We aim to provide data on the prevalence of HIV and sexually transmitted infection among PWIDS to inform national plans to get to zero.

Materials and Methods: Respondent-driven sampling was used to collect drug use, and sexual practices data among PWIDS aged 15 years and above. Blood samples were examined for HIV, herpes simplex virus type 2, syphilis, and hepatitis B.

Results: A total of 620 PWIDS with a median age of 32 (IQR17-52) participated in the study. Their use of drugs had typically started during adolescence. The prevalence of HIV was found to be $15.5 \%$ whereas that of herpes simplex type 2 was $43.3 \%$. Associated with an increased likelihood of HIV infection was being a female (adjusted odds ratio [aOR], 2.3, 95\%CI: 1.0-3.6), sharing of syringes (2.4, 95\%CI.1.1-6.1), used syringes hidden in public places (5.1, 95\%CI: 1.310.2) and having had a genital ulcer during the last 12 months before this survey. On the other hand, being educated, use of non-injectable drugs, access $(0.5,95 \%$ CI: $0.2-0.8)$ and use of clean syringes (0.3, 95\%CI: 0.1-0.6) were associated with decreased likelihood of HIV infection.

Conclusion: The prevalence of HIV infection among PWIDS in Dar es Salaam is three times higher than that in the general population. Behavioral and biological risk factors contribute to HIV transmission and needs to be addressed to be able to get to zero. 


\section{INTRODUCTION}

Around $10 \%$ of HIV infections globally are attributed to the use of injectable drugs [1-4]. A study covering 148 countries [4] showed that there were 3 million HIV positive persons among the 16 million people worldwide who were injecting drugs in 2008. However, HIV prevalence rates varied considerably between countries, ranging from $0.02 \%$ to $40 \%$. In Sub-Saharan Africa, which was estimated to be the home to 1.5 million injecting drug users, more than 200,000 were estimated to be HIV positive.

In Tanzania, previous studies have found high prevalence of HIV among people who inject drugs (PWIDS). In Zanzibar, the HIV prevalence was reported to be 16\% among PWIDS in 2007 [5], whereas $42 \%$ of PWIDS in Dar es Salaam were reported to be HIV positive in 2009 [6]. These estimates are now a few years old, and the overall HIV prevalence in the country has dropped in recent years (there was a decrease from $7.9 \%$ in 2003 to $5.1 \%$ in 2012 [7, 8]. In addition, several actors are now providing HIV prevention and harm reduction programming targeting PWIDS in Dar es Salaam, including needle and syringe programmes, medication-assisted treatment, and "sober houses" $[9,10]$. The decreasing HIV in the general population calls for intensified intervention for population at risk for HIV infection such as PWIDS to be able to achieve the global target of three zeros (zero new infection, zero discrimination and zero death). Taking stock of the current state of the HIV epidemic among PWIDS is an important step in the redesigning and scaling up of intervention measure. We present result from an integrated biobehavioral survey (IBBS) among PWIDS from Tanzania's largest urban area of Dar es Salaam. 


\section{MATERIAL AND METHODS}

\section{Study area}

This study was conducted in the three municipalities that make up the city of Dar es Salaam (Kinondoni, Ilala and Temeke) with a total population of about four million [11]. The overall HIV prevalence in the city is $9.3 \%$, considerably higher than the national average of 5.1\% [7]. Compared with the rest of the country, Dar es Salaam has been reported to have more drug use behaviors and a higher occurrence of sexual behaviors with risk for HIV transmission $[6,9,10]$.

\section{Study design and population}

This was a cross sectional study, conducted in 2015. All people 15 years and above residing in Dar es Salaam who had injected drugs during the past six months were eligible to be included. Residence was defined as having lived in the city the last six months preceding fieldwork.

\section{Sampling and sample size}

Respondent-driven sampling (RDS), a method developed for the sampling from populations for which there is no available sampling frame, was used to recruit study participants. RDS is based on the principle that members of the target population refer other members of the same population to participate, so that the sample is established by successive generations of recruitment referrals. RDS builds on a mathematical model which provides a theoretical basis for estimation of population proportions and their variances through statistical adjustment [12-13].

Sample size estimates were carried out with the aim to achieve $80 \%$ power to estimate the HIV prevalence among PWIDS. The estimation was based on the 2009 HIV prevalence study among PWIDS in Dar es Salaam where, as already mentioned, $42 \%$ were found to be HIV positive [6]. 
Taking into account a design effect of 1.8 due to respondent driven sampling (RDS), a total of 605 participants were found to be required given the mentioned assumptions.

\section{Data collection tools and administration}

The data collection instrument was in Swahili and was designed to take less than an hour to complete. Interview questions were developed in English and thereafter translated to Swahili by experienced translators before they were back-translated to English for validation purposes by the investigators (who are fluent in Swahili and familiar with terminology related to youth, health, HIV, substance/drug use, and sexual behavior).

After being informed about the study and having provided informed consent to participate in it, study participants took part in a structured interview administered in face to face conversations with trained interviewers. After completion of the interview, participants received pre-test counseling for HIV and STI. From those who agreed to testing, a blood sample was thereafter drawn for the testing of HIV, hepatitis B, syphilis, and herpes simplex virus type 2 (HSV 2).

\section{Laboratory testing}

HIV status was determined using Alere Determine ${ }^{\mathrm{TM}} \mathrm{HIV}-1 / 2$ assay (Alere Medical Co., Ltd, Japan). All samples that were reactive on the first assay were confirmed on a second rapid assay with Uni-Gold ${ }^{\mathrm{TM}}$ HIV-1/2 (Trinity Biotech Plc, Ireland). Discrepant results between the first and second rapid assays were resolved by Enzygnost HIV Integral II Antibody/Antigen ELISA (Siemens, Germany). Screening for the presence of hepatitis B surface antigen (HBsAg) was done using SD Bioline HBsAg rapid test (Standard Diagnostics, Inc., Korea) and reactive samples were confirmed on a microparticle enzyme immune-assay (MEIA) (Abbott AxSYM, Germany). Syphilis screening was performed using the Venereal Disease Research Laboratory 
assay (VDRL; Omega Diagnostic, UK) and reactive samples were confirmed by Treponemal Particle Hemagglutination Assay (TPHA; Omega Diagnostic, UK). Herpes simplex virus type 2 (HSV-2) serostatus was determined using HSV-2 IgG ELISA (Abbott Murex, UK) and reactive specimens were further tested with HSV-2 IgM ELISA to exclude active infection.

\section{Ethical considerations}

Ethical approval was obtained from the Research and Publication Committee of the Muhimbili University of Health and Allied Sciences, and permission to conduct the study was thereafter obtained from the municipal authorities. To ensure confidentiality, interviews were conducted in private and secure places chosen by the participants themselves. Written informed consent was obtained from participants before the interview commenced. Health education regarding safe injection practices and safer sex was provided to all study participants, and those who tested positive for HIV and/or an STI were referred to a care and treatment clinic.

\section{Data analysis}

Data were entered in SPSS version 15, whereas analyses were carried out using the STATA and RDSAT statistical packages. Since the selection probability is not the same for each participant when RDS is applied (larger networks are more likely to be represented than those that are smaller), data were weighted by calculating weight as an inverse of the participant's network size [13]. To reduce clustering and ensure that the whole sample was reflected in the analysis, we multiplied the weight by the sample size and divided it by the sum of the weights. Frequencies were calculated to show the proportional distribution of categorical variables. Mean and standard deviation was used to summarize continuous variables. Logistic regression models were built to identify independent predictors of HIV infection. All analyses were two-tailed and the significance level was set at $5 \%$. 


\section{RESULTS}

A total of 620 persons participated in this study, with males constituting a large majority (94.4\%) of the sample. As shown in Table 1, participants' age ranged from 17 to 52 years, and the median age was 32 years. More than half (53.1\%) had completed primary school, and higher educational level than this was comparatively rare (only $7.6 \%$ had secondary school education or higher).

More than two-thirds (68\%) of the participants were single, whereas $13.7 \%$ were cohabiting with a partner, $11.6 \%$ were divorced or separated, and $5.7 \%$ were married. Slightly more than half (55.5\%) had one or more children. Almost a third (30.4\%) were living alone at the time of the survey, whereas $14.7 \%$ were living with a spouse or partner, twenty per cent were living with parents, and $18 \%$ were staying with other relatives.

The reported monthly income varied from 100 to 3,000,000 Tanzanian shillings (i.e., from 5 US cents to 1,400 USD), with the median being 300,000 shillings (140 USD). Self-employment was reported as the main occupation by $37.1 \%$, and $9 \%$ were petty traders.

\section{Drug use}

About one out of every ten (11.4\%) study participants had started to inject drugs when they were 15 years of age or younger, whereas 54.5\% had debuted while they were between 15 and 24 years old. A majority (62.9\%) said they had been introduced to injection practices by a friend.

During the last three months before the interview, 57.5\% reported having injected white heroine, $3.9 \%$ said they had injected brown heroine, $6.2 \%$ had taken cocaine, and $0.2 \%$ had used prescription drugs.

A large majority (96.2\%) said that access to clean syringes and needles was not a problem for them, and drug stores were the most common source of injection paraphernalia. Use of needles 
or syringes that had been used by someone else was reported by $12.5 \%$. Almost a third (31.9\%) had a history of joint preparation of drugs before use.

\section{Sexual practices}

The age at first sexual experience ranged from 7 to 28 years. The median was 18 years, and about a quarter of the study participants had been sexually active by the time they reached 15 years of age. A large majority $(90.1 \%)$ had ever used a condom, and more than half (51.8\%) said it was fairly easy to get male condoms when needed. The majority of respondents $(77.8 \%)$ said they bought condoms from shops. Other sources of condom supply included drug stores (4.2\%), health facilities (7.4\%), and bars, guest houses and hotels $(6.2 \%)$.

\section{Self-assessment of risk for HIV infection}

Almost two out of every five $(38.7 \%)$ respondents considered themselves to be at no or low risk of HIV infection. Males were more likely to report no or low risk than females $(40 \%$ versus $29 \%, \mathrm{p}<0.001)$. Of all the participants, $71.1 \%$ reported to have ever tested for HIV and of these, $55.8 \%$ had tested during the past year preceding the survey. Moreover, of those who tested for HIV, 66.5\% received post-test counseling and were aware of their HIV status. Prior HIV testing did not differ by sex $(\mathrm{p}=0.427)$.

\section{Prevalence of HIV and selected sexually transmitted infections}

Of the 620 participants who participated in this survey, $610(98.4 \%)$ provided blood for testing of HIV and other sexually transmitted infections. The HIV prevalence was $15.5 \%$ and that of herpes simplex virus type 2 was $43.3 \%$. The prevalence of hepatitis B was $1.1 \%$, whereas none of the study participants had syphilis. 
As shown in Table 2, HIV prevalence was associated with low education level, syringe sharing, syringe re-use, and having had a genital ulcer during the last 12 months preceding the survey.

\section{Independent predictors of HIV infection}

A multiple logistic regression model was built to examine for independent predictors of HIV. As shown in Table 3, women who inject drugs were considerably more likely than men to have an HIV infection.

Female PWIDS were two times more likely to be HIV seropositive when compared to one (AOR, 2.3, 95\% CI: 1.0-3.6).

Higher educational level was associated with a lower occurrence of HIV. PWIDS who reported to have completed primary school were $60 \%$ less likely to be infected with HIV as compared to those who had never gone to school, whereas those with higher than primary education were $70 \%$ less likely to be seropositive.

PWIDS who reported to have used non-injectable drugs during the past 12 months had $40 \%$ lower likelihood of being HIV infected as compared to those who did not report use of such drugs. Access to clean and safe syringes (AOR, 0.5, 95\% CI: 0.2-0.8) or cleaning of syringes (AOR, 0.3, 95\%CI: 0.1-0.6) during sharing was associated with decreased risk of HIV infection in this population. On the other hand, sharing of syringe (AOR, 2.4, 95\%CI: 1.1-6.1), using used syringe hidden in public places (AOR, 5.1, 95\%CI: 1.3-10.2) and/or having a history of genital ulcer during the past 12 months preceding the survey (AOR, 4.8, 95\% CI: 1.4-12.6) were associated with increased likelihood of HIV infection 


\section{DISCUSSION}

This is the largest integrated bio-behavioral survey that has been conducted among people who inject drugs in Tanzania to date. It provides the most recent estimates of the prevalence of HIV and selected sexually transmitted infections, and some of their attendant risks, in this segment of the Tanzanian population.

A main finding is that PWIDS in Dar es Salaam continue to carry a very significant burden of HIV, and currently have an HIV prevalence that is three times higher than that in the overall Tanzanian population (15.5\% versus 5.1\%) [7]. At a time when the overall rate of HIV infections is on the decrease in the country $[7,8]$, these findings indicate that there is still a strong rationale for HIV programming that engages thoroughly and inclusively with population subgroups that suffer the most dramatic consequences of the HIV epidemic, such as PWIDS to be able to achieve the global agreed target of zero new infection.

While the presented HIV prevalence estimates are high, they are considerably lower than the overall prevalence of $42 \%$ reported among PWIDS in Dar es Salaam in 2009 [6]. While it is not possible to determine the reasons for this difference on the basis of this cross-sectional study alone, part of the explanation is undoubtedly the unalike sex composition of the two study populations. In the 2009 survey, $41 \%$ of study participants were women, whereas the proportion of women in this study was 5.6\% (this was the outcome of respondent-driven sampling, and should in theory reflect the population proportions of women who inject drugs in Dar es Salaam). As we have shown, HIV prevalence is considerably higher among female than among male PWIDS in Dar es Salaam, and the inclusion of proportionally more women in the 2009 study in part explains the higher overall prevalence estimate reported by that study. Had the sex ratio 
been the same in the current study as in the mentioned 2009 survey, the overall average HIV prevalence in our study would have been $19.2 \%$.

Even when the differences produced by the dissimilar sex composition in the two studies are taken into consideration in this way, however, the HIV prevalence among PWIDS is currently only about half of what it was seven years ago. Although some of the difference could conceivably be ascribed to an overestimate in the 2009 study, and to methodological differences between the two studies, it would seem possible that this large difference could reflect a genuine decrease in HIV prevalence among people who inject drugs in Dar es Salaam. There has been a considerable build-up of HIV prevention and care programming with and among PWIDS in the city in recent years, and several projects in town now provide health education as well as harm reduction services [9]. Aided also by a concurrent general decrease in HIV prevalence in the Tanzanian population in recent years, it is possible that this study provides the first indication that the impact of these interventions are about to come into effect.

Another significant finding of this study was the high prevalence of HSV-2 in the study population. It is well known from biological as well as epidemiological research that herpes simplex infections facilitate HIV transmission and acquisition [14-16]. Moreover, since HSV-2 is transmitted sexually, but not through the sharing of injection equipment, it is often used as a biomarker for sexual HIV transmission among PWIDS [17]. As compared to many studies all over Africa, most of the participants in this study reported to have access to clean syringes and needles. This finding could be attributed to ongoing syringe and needles provision program run by Medicine du Monde in the City. Given the increasing focus on safe injection practices in prevention programming with PWIDS in Dar es Salaam, and the good availability of needles and syringes reported by the study participants themselves, the high HSV-2 prevalence may suggest 
that sexual transmission may now be playing an important role in the HIV epidemic among PWIDS in the city. Moreover, a history of genital ulcer, probably in most cases due to HSV-2 infections, was associated with five times higher odds of HIV infection in this population.

When asked, a large proportion of study participants assessed their own risk for HIV acquisition or transmission as low. PWIDS clearly seem to underestimate their own general risk for HIV infection. This is consistent with the findings of other recent research that has found that there is a mismatch between risk behaviors among PWIDS in Dar es Salaam and screening for HIV infection [18]. The under-estimation of own risk is important to take into consideration in the design of intervention measures and monitoring of behavioral change intervention in the future.

The overall findings of this study adds to the rationale for HIV programming in Tanzania that focuses on high risk groups such as PWIDS. The results also suggest that prevention of HIV infection among PWIDS needs to address both injection practices and risky sexual behaviors.

A substantial proportion of participants $(71.1 \%)$ reported to have ever tested for HIV and $66.5 \%$ retuned for post-test counseling and knew their HIV status. These results indicate that PWIDS in Dar es Salaam have relatively good access to HIV related services and this could be an opportunity for delivery of targeted HIV prevention messages. In this study, all participants who tested positive for HIV were referred to a nearby care and treatment facility and were enrolled to care and treatment.

The findings of this study should be interpreted in light of the following limitations. Firstly, this was a cross sectional study which has the inherent weakness of entangling cause and effect relationships. However, the predictors of HIV infection we have studied are both biologically plausible and have been proven by robust epidemiological studies earlier. Secondly; drug use and 
sexual practices may be perceived as sensitive issues, and our study may therefore have suffered from desirability bias. This bias may have been more likely to underestimate rather than to overestimate risks. Lastly, this study was conducted in Dar es Salaam and the results may not be representative of PWIDS elsewhere in Tanzania. However, due the fact that large proportion of injecting drug use are in this city, intervention targeting PWIDS in Dar es Salaam are more likely to impact HIV transmission in a larger extent.

\section{CONCLUSIONS}

People who inject drugs in Dar es Salaam continue to carry a considerable burden of HIV and have an HIV prevalence that is three times larger than that in the overall population. At the same time, this study is the first to suggest that the HIV epidemic among PWIDS may currently be on the decline in Dar es Salaam, possibly reflecting the impact of HIV prevention programming with and among PWIDS. The prevalence of HSV-2 is very high and may suggest that sexual transmission of HIV is playing a central role in HIV transmission among PWIDS. A substantial proportion of PWIDS perceive themselves to be at no or low risk of HIV infection. To be able to achieve the global goals of zero new HIV infections and zero deaths from HIV, ongoing HIV prevention programming targeting PWIDS should be continued and strengthened. Behavioral intervention through peer outreach, medically assisted therapy such as use of methadone, syringes and needles support, targeted HIV testing and counselling should be given priority. This will be in line with the implementation of the available comprehensive guidelines for HIV prevention among key populations in Tanzania. 


\section{REFERENCES}

1. Mathers BM, Degenhardt L, Phillips B, etal. Global epidemiology of injecting drug use and HIV among people who inject drugs: A systematic review. Lancet, 2008, 372:17331745.

2. Wabwire-Mangen F. Uganda HIV modes of transmission and prevention response analysis. Kampala, Uganda National AIDS Commission, 2009

3. Aceijas C. Global overview of injecting drug use and HIV infection among injecting drug users. AIDS 2004;18(17):2295-303.

4. Strathdee SA, Stockman JK. Epidemiology of HIV Among Injecting and Non-Injecting Drug Users: Current Trends and Implications for Intervention. Curr HIV/AIDS Rep, 2010; 7(2): 99-106

5. Dahoma MJ, Salim AA, Abdool R et al. HIV and substance abuse: the dual epidemics challenging Zanzibar. African Journal of Drug and Alcohol Studies, 2006, 5:129-138.

6. Williams ML, McCurdy SA, Bowen AM et al. HIV seroprevalence in a sample of Tanzanian intravenous drug users. AIDS Educ Prev. 2009 Oct;21(5):474-83.

7. Tanzania Commission for AIDS (TACAIDS), Zanzibar AIDS Commission (ZAC), national Bureau of Statistics (NBS), Office of the Chief Government Statistician (OCGS), ICF International Tanzania HIV/AIDS and Malaria Indicator Survey 2011 2012. Dar es salaam, Tanzania: (2013). TACAIDS, ZAC, NBS, OCGS, ICF International

8. Tanzania Commission for AIDS (TACAIDS), Zanzibar AIDS Commission (ZAC), national Bureau of Statistics (NBS), Office of the Chief Government Statistician 
(OCGS), ICF International Tanzania HIV/AIDS and Malaria Indicator Survey 2007/8. Dar es salaam, Tanzania: (2008). TACAIDS, ZAC, NBS, OCGS, ICF International

9. Ratliff EA, McCurdy SA, Mbwambo JK et al. An overview of HIV prevention interventions for people who inject drugs in Tanzania. Adv Prev Med. 2013; 2013:183187

10. Ross MW, McCurdy SA, Kilonzo GP et al. Drug use careers and blood-borne pathogen risk behavior in male and female Tanzanian heroin injectors. Am J Trop Med Hyg. 2008 Sep;79(3):338-43.

11. National Bureau of Statistics (NBS). National Population Census 2012. Dar es Salaam, Tanzania: NBS Tnzania

12. Heckthorn DD. Respondents driven sampling. A new approach to the study of Hidden populations. Soc Problems 2008; 44:174-99.

13. Wejnert C. An emprerical test of respondent-driven sampling: Point estimates, variance, degree measures, and out-of equilibrium data. Sociol methodol 2009; 39:73-116.

14. Freeman EE, Weiss HA, Glynn JR et al. Herpes simplex virus 2 infection increases HIV acquisition in men and women: systematic review and meta-analysis of longitudinal studies. AIDS. 2006;20(1):73-83

15. Tobian A, Quinn T. Herpes simplex virus type 2 and syphilis infections with HIV: an evolving synergy in transmission and prevention. Curr Opin HIV AIDS. 2009;4(4):294299

16. Wald A, Link K. Risk of human immunodeficiency virus infection in herpes simplex virus type 2-seropositive persons: a meta-analysis. J Infect Dis. 2002;185(1):45-52

17. Des Jarlais D, Arasteh $\mathrm{K}$, McKnight $\mathrm{C}$ et al. Using hepatitis $\mathrm{C}$ virus and herpes simplex 
virus-2 to track HIV among injecting drug users in New York City. Drug Alcohol Depend. 2009;101(1-2):88-91

18. Mlunde LB, Sunguya BF, Mbwambo JK et al. Mismatch between High-Risk Behaviors and Screening of Infectious Diseases among People Who Inject Drugs in Dar es Salaam, Tanzania. PLoS One. 2016 Feb 5;11(2): e0148598 
Table 1: Demographic characteristics of people who inject drugs in Dar es Salaam, Tanzania

\begin{tabular}{|c|c|c|}
\hline Variable & & $\% \quad \mathrm{~N}=620$ \\
\hline \multirow[t]{2}{*}{ Sex } & Males & 94.4 \\
\hline & Females & 5.6 \\
\hline \multirow[t]{3}{*}{ Age } & Youth (Age range) & 11.5 \\
\hline & Young adults & 55.0 \\
\hline & Middle age & 33.5 \\
\hline \multirow[t]{5}{*}{ Level of education } & No formal education & 6.7 \\
\hline & Incomplete primary education & 16.9 \\
\hline & Primary education & 53.8 \\
\hline & Incomplete secondary education & 14.1 \\
\hline & Secondary education of higher & 7.6 \\
\hline \multirow[t]{5}{*}{ Marital Status } & Single & 68 \\
\hline & Married & 5.7 \\
\hline & Separated/divorced & 11.6 \\
\hline & Widowed/widower & 0.7 \\
\hline & Cohabiting & 13.9 \\
\hline \multirow[t]{7}{*}{ Currently living with } & Alone & 30.4 \\
\hline & Spouse/partner & 14.7 \\
\hline & Partner & 8.2 \\
\hline & Parents & 20.0 \\
\hline & Other relatives & 18.0 \\
\hline & Friends & 3.5 \\
\hline & No fixed residence & 5.1 \\
\hline
\end{tabular}


Table 2: HIV prevalence by Risk factors for HIV transmission among PWIDS in Dar es Salaam, Tanzania

\begin{tabular}{|c|c|c|c|c|}
\hline Variable & categories & $\mathrm{n}$ & $\% \mathrm{HIV+}$ & P-value \\
\hline \multirow[t]{3}{*}{ Age group (years) } & $15-24$ & 22 & 12.7 & 0.790 \\
\hline & $25-34$ & 44 & 15.3 & \\
\hline & 35 and above & 30 & 16.3 & \\
\hline \multirow[t]{2}{*}{ Sex } & Male & 72 & 14.8 & 0.001 \\
\hline & Female & 24 & 25.5 & \\
\hline \multirow[t]{4}{*}{ Education level } & No education & 39 & 37.1 & 0.002 \\
\hline & Primary incomplete & 44 & 15.2 & \\
\hline & Primary complete & 13 & 11.1 & \\
\hline & Above primary & 12 & 8.9 & \\
\hline \multirow[t]{3}{*}{ Marital status } & Single & 53 & 15.6 & 0.477 \\
\hline & Married/cohabiting & 20 & 11.4 & \\
\hline & Divorced/separated & 23 & 10.2 & \\
\hline \multirow[t]{2}{*}{ Live with spouse } & No & 50 & 16.7 & 0.901 \\
\hline & yes & 46 & 16.0 & \\
\hline \multirow[t]{2}{*}{ Have your own kids } & No & 49 & 16.5 & 0.777 \\
\hline & yes & 47 & 16.0 & \\
\hline Used non-injectable drug past 12 & No & 35 & 15.1 & 0.081 \\
\hline months & Yes & 61 & 10.5 & \\
\hline \multirow[t]{2}{*}{ Syringe access when needed } & No & 26 & 16.7 & 0.895 \\
\hline & Yes & 70 & 15.5 & \\
\hline
\end{tabular}




\begin{tabular}{|c|c|c|c|c|}
\hline \multirow[t]{2}{*}{ Shared syringe past 3 months } & No & 39 & 11.0 & 0.046 \\
\hline & Yes & 57 & 14.0 & \\
\hline \multirow[t]{2}{*}{ Used syringe hidden in a public place } & No & 44 & 14.9 & 0.028 \\
\hline & Yes & 52 & 23.3 & \\
\hline \multirow[t]{2}{*}{ Cleaned syringe when sharing } & No & 14 & 30.4 & 0.014 \\
\hline & Yes & 82 & 12.0 & \\
\hline \multirow[t]{2}{*}{ Ever had anal sex past 12 months } & No & 28 & 20.5 & 0.092 \\
\hline & yes & 68 & 18.3 & \\
\hline \multirow[t]{2}{*}{ Ever used condom } & No & 23 & 20.0 & 0.054 \\
\hline & Yes & 73 & 15.1 & \\
\hline \multirow[t]{2}{*}{ Had genital discharge past 12 months } & No & 60 & 13.4 & 0.920 \\
\hline & Yes & 36 & 12.3 & \\
\hline \multirow[t]{2}{*}{ Had genital ulcers past 12 months } & No & 57 & 11.9 & 0.005 \\
\hline & Yes & 39 & 27.0 & \\
\hline \multirow[t]{2}{*}{ Ever experienced violence/abuse } & No & 51 & 15.6 & 0.583 \\
\hline & Yes & 45 & 15.2 & \\
\hline
\end{tabular}


Table 3: Multiple Regression analysis of independent predictors of HIV infection among PWIDS in Dar es Salaam, Tanzania

\begin{tabular}{|c|c|c|c|}
\hline Variable & Categories & AOR (95\% CI) & p- value \\
\hline \multirow[t]{3}{*}{ Age group (years) } & $15-24$ & 1 & \\
\hline & $25-34$ & $0.9(0.3-2.3)$ & 0.855 \\
\hline & 35 and above & $0.7(0.2-1.9)$ & 0.500 \\
\hline \multirow[t]{2}{*}{ Sex } & Male & 1 & \\
\hline & Female & $2.3(1.0-3.6)$ & 0.034 \\
\hline \multirow[t]{4}{*}{ Education level } & No education & 1 & \\
\hline & Primary incomplete & $0.5(0.1-1.0)$ & 0.061 \\
\hline & Primary complete & $0.4(0.2-0.9)$ & 0.008 \\
\hline & Above primary & $0.3(0.1-0.7)$ & 0.004 \\
\hline \multirow[t]{3}{*}{ Marital status } & Single & 1 & \\
\hline & Married/cohabiting & $1.0(0.2-3.9)$ & 0.953 \\
\hline & Divorced/separated & $1.3(0.4-3.7)$ & 0.550 \\
\hline \multirow[t]{2}{*}{ Live with spouse } & No & 1 & \\
\hline & yes & $0.9(0.4-1.8)$ & 0.863 \\
\hline \multirow[t]{2}{*}{ Have your own kids } & No & 1 & \\
\hline & yes & $1.1(0.8-2.3)$ & 0.912 \\
\hline Ever used non injectable drugs past 12 & No & 1 & \\
\hline months & Yes & $0.6(0.3-0.9)$ & 0.037 \\
\hline \multirow[t]{2}{*}{ Syringe access when needed } & No & 1 & \\
\hline & Yes & $0.5(0.2-0.8)$ & 0.008 \\
\hline Shared syringe past 3 months & No & 1 & \\
\hline
\end{tabular}




$$
\text { Yes }
$$

0.004

Used syringe hidden in a public place

No

1

Yes

5.1(1.3-10.2) $\quad 0.002$

Cleaned syringe when sharing

No

1

Yes

$0.3(0.1-0.6)$

$<0.001$

Ever had anal sex past 12 months

No

1

yes

1.2(0.6-4.3) $\quad 0.316$

Ever used condom

No

1

Yes

$0.7(0.2-1.2) \quad 0.054$

Had genital discharge past 12 months

No

1

Yes

2.1(0.9-7.1) $\quad 0.062$

Had genital ulcers past 12 months

No

1

Yes

4.8(1.4-12.6) $\quad 0.006$

Ever experienced violence/abuse

No

1

Yes

$0.8(0.3-5.6)$

0.871 